\title{
WEIGHTED SHARING OF THREE VALUES AND UNIQUENESS OF MEROMORPHIC FUNCTIONS
}

\author{
INDRAJIT LAHIRI
}

\begin{abstract}
Using the idea of weighted sharing we prove a result on uniqueness of meromorphic functions sharing three values which improve some results of Ueda, Yi and Ye.
\end{abstract}

\section{Introduction, definitions and results}

Let $f$ and $g$ be two nonconstant meromorphic functions defined in the open complex plane $\mathscr{C}$. For $b \in \mathscr{C} \cup\{\infty\}$ we say that $f$ and $g$ share the value $b \mathrm{CM}$ (counting multiplicities) if $f-b$ and $g-b$ have the same zeros with the same multiplicities. If we do not take multiplicities into account, we say that $f$ and $g$ share the value $b$ IM (ignoring multiplicities). For standard notations and definitions of the value distribution theory we refer [1].

H. Ueda [6] proved the following result.

THEOREM A [6]. Let $f$ and $g$ be two distinct nonconstant entire functions sharing $0,1 \mathrm{CM}$ and let $a(\neq 0,1)$ be a finite complex number. If a is lacunary for $f$ then $1-a$ is lacunary for $g$ and $(f-a)(g+a-1) \equiv a(1-a)$.

Improving Theorem A H. X. Yi [8] proved the following theorem.

THEOREM B [8]. Let $f$ and $g$ be two distinct nonconstant entire functions sharing $0,1 \mathrm{CM}$ and let $a(\neq 0,1)$ be a finite complex number. If $\delta(a ; f)>1 / 3$ then $a$ and $1-a$ are Picard exceptional values of $f$ and $g$ respectively and $(f-a)(g+a-1) \equiv a(1-a)$.

Extending Theorem B to meromorphic functions S. Z. Ye [7] proved the following results.

THEOREM C [7]. Let $f$ and $g$ be two distinct nonconstant meromorphic functions such that $f$ and $g$ share $0,1, \infty \mathrm{CM}$. Let $a(\neq 0,1)$ be a finite complex

2000 Mathematics Subject Classification: 30D35.

Key words and phrases: Meromorphic function, weighted sharing, uniqueness.

Received October 12, 2000; revised April 9, 2001. 
number. If $\delta(a ; f)+\delta(\infty ; f)>4 / 3$ then $a$ and $1-a$ are Picard exceptional values of $f$ and $g$ respectively and also $\infty$ is so and $(f-a)(g+a-1) \equiv a(1-a)$.

THEOREM D [7]. Let $f$ and $g$ be two distinct nonconstant meromorphic functions sharing $0,1, \infty \mathrm{CM}$. Let $a_{1}, a_{2}, \ldots, a_{p}$ be $p(\geq 1)$ distinct finite complex numbers and $a_{j} \neq 0,1$ for $j=1,2,3 \ldots p$. If $\sum_{j=1}^{p} \delta\left(a_{j} ; f\right)+\delta(\infty ; f)>2(p+1) /$ $(p+2)$ then there exist one and only one $a_{k}$ in $a_{1}, a_{2}, \ldots, a_{p}$ such that $a_{k}$ and $1-a_{k}$ are Picard exceptional values of $f$ and $g$ respectively and also $\infty$ is so and $\left(f-a_{k}\right)\left(g+a_{k}-1\right) \equiv a_{k}\left(1-a_{k}\right)$.

Improving above results $\mathrm{H}$. X. Yi [10] proved the following theorem.

THEOREM E [10]. Let $f$ and $g$ be two distinct nonconstant meromorphic functions such that $f$ and $g$ share $0,1, \infty \mathrm{CM}$. Let $a(\neq 0,1)$ be a finite complex number. If $N(r, a ; f) \neq T(r, f)+S(r, f)$ and $N(r, f) \neq T(r, f)+S(r, f)$ then $a$ and $1-a$ are Picard exceptional values of $f$ and $g$ respectively and also $\infty$ is so and $(f-a)(g+a-1) \equiv a(1-a)$.

Definition 1. Let $p$ be a positive integer and $b \in \mathscr{C} \cup\{\infty\}$. Then by $N(r, b ; f \mid \leq p)$ we denote the counting function of those zeros of $f-b$ (counted with proper multiplicities) whose multiplicities are not greater than $p$. By $\bar{N}(r, b ; f \mid \leq p)$ we denote the corresponding reduced counting function.

In an analogous manner we define $N(r, b ; f \mid \geq p)$ and $\bar{N}(r, b ; f \mid \geq p)$.

Hua and Fang [2] proved that if two nonconstant distinct meromorphic functions $f$ and $g$ share $0,1, \infty \mathrm{CM}$ then $N(r, a ; f \mid \geq 3)=S(r, f)$ for any complex number $a(\neq 0,1, \infty)$.

Also $\mathrm{Yi}$ [10] proved that if two nonconstant distinct meromorphic functions $f$ and $g$ share $0,1, \infty \mathrm{CM}$ then $N(r, \infty ; f \mid \geq 2)=S(r, f)$.

Therefore Theorem $\mathrm{E}$ of $\mathrm{Yi}$ can easily be improved to the following result.

THEOREM 1. Let $f$ and $g$ be distinct nonconstant meromorphic functions sharing $0,1, \infty \mathrm{CM}$. If $a(\neq 0,1)$ is a finite complex number such that $N(r, a ; f \mid \leq 2) \neq T(r, f)+S(r, f)$ and $N(r, \infty ; f \mid \leq 1) \neq T(r, f)+S(r, f)$ then $a$ and $1-a$ are Picard exceptional values of $f$ and $g$ respectively and also $\infty$ is so and $(f-a)(g+a-1) \equiv a(1-a)$.

Following examples show that Theorem 1 is sharp.

Example 1. Let $f=\left(e^{z}-1\right) /\left(e^{z}+1\right), g=\left(1-e^{z}\right) /\left(1+e^{z}\right), a_{1}=-1$ and $a_{2}=2$. Then $f, g$ share $0,1, \infty$ CM. Also $N(r, \infty ; f \mid \leq 1)=T(r, f)+S(r, f)$, $N\left(r, a_{1} ; f \mid \leq 2\right) \neq T(r, f)+S(r, f)$ and $N\left(r, a_{2} ; f \mid \leq 2\right)=T(r, f)+S(r, f)$. Clearly $\left(f-a_{i}\right)\left(g+a_{i}-1\right) \not \equiv a_{i}\left(1-a_{i}\right)$ for $i=1,2$.

Example 2. Let $f=e^{z}, g=e^{-z}$ and $a=2$. Then $f, g$ share $0,1, \infty \mathrm{CM}$. 
Also $N(r, \infty ; f \mid \leq 1) \neq T(r, f)+S(r, f), \quad N(r, a ; f \mid \leq 2)=T(r, f)+S(r, f)$. Clearly $(f-a)(g+a-1) \not \equiv a(1-a)$.

Now one may ask the following question: Is it possible to replace the hypothesis $N(r, a ; f \mid \leq 2) \neq T(r, f)+S(r, f)$ of Theorem 1 by any one of the following?

(i) $N(r, a ; f \mid \leq 1) \neq T(r, f)+S(r, f)$,

(ii) $\bar{N}(r, a ; f \mid \leq 2) \neq T(r, f)+S(r, f)$.

We can answer this question in the negative by the following example.

Example 3. Let $f=e^{z}\left(1-e^{z}\right), g=e^{-z}\left(1-e^{-z}\right)$ and $a=1 / 4$. Then $f, g$ share $0,1, \infty$ CM. Also $N(r, \infty ; f \mid \leq 1) \neq T(r, f)+S(r, f)$. Since $f-a=$ $-\left(e^{z}-2 a\right)^{2}$, we see the following

(i) $N(r, a ; f \mid \leq 1) \equiv 0$,

(ii) $\bar{N}(r, a ; f \mid \leq 2)=N\left(r, 2 a ; e^{z}\right)=(1 / 2) T(r, f)+S(r, f)$ and

(iii) $N(r, a ; f \mid \leq 2)=2 N\left(r, 2 a ; e^{z}\right)=T(r, f)+S(r, f)$.

Also clearly $(f-a)(g+a-1) \not \equiv a(1-a)$.

First we note that if $f, g$ satisfy the conclusion of the theorems as stated above then $f, g$ must share $\infty \mathrm{CM}$ because in this case $\infty$ becomes lacunary for $f$ and $g$ and so the question of sharing $\infty$ IM does not arise.

Now the following two examples show that in the above theorems the sharing of 0 and 1 can not be relaxed from CM to IM.

Example 4. Let $f=e^{z}-1, g=\left(e^{z}-1\right)^{2}$ and $a=-1$. Then $f, g$ share 0 IM and $1, \infty$ CM. Also $N(r, \infty ; f) \equiv 0$ and $N(r, a ; f) \equiv 0$ but $(f-a)(g+a-1) \not \equiv$ $a(1-a)$.

Example 5. Let $f=2-e^{z}, g=e^{z}\left(2-e^{z}\right)$ and $a=2$. Then $f, g$ share 1 IM and $0, \infty$ CM. Also $N(r, \infty ; f) \equiv 0$ and $N(r, a ; f) \equiv 0$ but $(f-a)(g+a-1) \not \equiv$ $a(1-a)$.

Now one may ask the following question: Is it really impossible to relax in any way the nature of sharing of any one of 0 and 1 in the theorems stated above?

In the paper we study this problem. Though we do not know the situation for Theorem 1 we can relax the nature of sharing of 0 and 1 separately in Theorem C and thereby we can improve Theorem A, Theorem B and Theorem C. $[4,5]$.

To this end we now explain the notion of weighted sharing as introduced in

Definition $2[4,5]$. Let $k$ be a nonnegative integer or infinity. For $a \in \mathscr{C} \cup$ $\{\infty\}$ we denote by $E_{k}(a ; f)$ the set of all $a$-points of $f$ where an $a$-point of multiplicity $m$ is counted $m$ times if $m \leq k$ and $k+1$ times if $m>k$. If $E_{k}(a ; f)=E_{k}(a ; g)$, we say that $f, g$ share the value a with weight $k$.

The definition implies that if $f, g$ share a value $a$ with weight $k$ then $z_{o}$ is a zero of $f-a$ with multiplicity $m(\leq k)$ if and only if it is a zero of $g-a$ with 
multiplicity $m(\leq k)$ and $z_{o}$ is a zero of $f-a$ with multiplicity $m(>k)$ if and only if it is a zero of $g-a$ with multiplicity $n(>k)$ where $m$ is not necessarily equal to $n$.

We write $f, g$ share $(a, k)$ to mean that $f, g$ share the value $a$ with weight $k$. Clearly if $f, g$ share $(a, k)$ then $f, g$ share $(a, p)$ for all integer $p, 0 \leq p<k$. Also we note that $f, g$ share a value $a$ IM or CM if and only if $f, g$ share $(a, 0)$ or $(a, \infty)$ respectively.

Definition 3 [4]. For $S \subset \mathscr{C} \cup\{\infty\}$, we define $E_{f}(S, k)$ as $E_{f}(S, k)=$ $\bigcup_{a \in S} E_{k}(a ; f)$, where $k$ is a nonnegative integer or infinity.

DeFINITION 4. For $a \in \mathscr{C} \cup\{\infty\}$, we put

$$
\delta_{p)}(a ; f)=1-\limsup _{r \rightarrow \infty} \frac{N(r, a ; f \mid \leq p)}{T(r, f)},
$$

where $p$ is a positive integer.

Now we state the main results of the paper.

THEOREM 2. Let $f$ and $g$ be two distinct meromorphic functions sharing $(0,1)$, $(1, \infty)$ and $(\infty, \infty)$. If $a(\neq 0,1)$ is a finite complex number such that $3 \delta_{2)}(a ; f)+$ $2 \delta_{1)}(\infty ; f)>3$ then $a$ and $1-a$ are Picard exceptional values of $f$ and $g$ and also $\infty$ is so and $(f-a)(g+a-1) \equiv a(1-a)$.

THEOREM 3. Let $f$ and $g$ be two distinct meromorphic functions sharing $(0, \infty)$, $(1,1)$ and $(\infty, \infty)$. If $a(\neq 0,1)$ is a finite complex number such that $\left.3 \delta_{2}\right)(a ; f)+$ $2 \delta_{1)}(\infty ; f)>3$ then $a$ and $1-a$ are Picard exceptional values of $f$ and $g$ and also $\infty$ is so and $(f-a)(g+a-1) \equiv a(1-a)$.

Example 4 shows that in Theorem 2 sharing $(0,1)$ can not be relaxed to sharing $(0,0)$ and Example 5 shows that in Theorem 3 sharing $(1,1)$ can not be relaxed to sharing $(1,0)$.

Throughout the paper we denote by $f, g$ two nonconstant meromorphic functions defined in the open complex plane $\mathscr{C}$.

\section{Lemmas}

In this section we present some lemmas which will be required in the sequel.

LEMma 1. If $f$ and $g$ share $(0,0),(1,0)$ and $(\infty, 0)$ then

$$
\text { (i) } T(r, f) \leq 3 T(r, g)+S(r, f)
$$

and

$$
\text { (ii) } T(r, g) \leq 3 T(r, f)+S(r, g) \text {. }
$$

Proof. Since $f, g$ share $(0,0),(1,0)$ and $(\infty, 0)$, by the second fundamental theorem we get 


$$
\begin{aligned}
T(r, f) & \leq \bar{N}(r, 0 ; f)+\bar{N}(r, 1 ; f)+\bar{N}(r, \infty ; f)+S(r, f) \\
& =\bar{N}(r, 0 ; g)+\bar{N}(r, 1 ; g)+\bar{N}(r, \infty ; g)+S(r, g) \\
& \leq 3 T(r, g)+S(r, f),
\end{aligned}
$$

which is (i).

Similarly we can prove (ii). This proves the lemma.

Lemma 2. Let $f$ and $g$ share $(0,1),(1, \infty),(\infty, \infty)$ and $f \not \equiv g$. Then

(i) $\bar{N}(r, 0 ; f \mid \geq 2)+N(r, \infty ; f \mid \geq 2)+N(r, 1 ; f \mid \geq 2)=S(r, f)$,

(ii) $\bar{N}(r, 0 ; g \mid \geq 2)+N(r, \infty ; g \mid \geq 2)+N(r, 1 ; g \mid \geq 2)=S(r, f)$.

Proof. We prove (i) because (ii) follows from (i) since $f$ and $g$ share $(0,1)$, $(1, \infty),(\infty, \infty)$.

First we show that $\bar{N}(r, 0 ; f \mid \geq 2)=S(r, f)$. If $\bar{N}(r, 0 ; f)=S(r, f)$ then there is nothing to prove. So we suppose that $\bar{N}(r, 0 ; f) \neq S(r, f)$. Let

$$
\phi=\frac{f^{\prime}}{f-1}-\frac{g^{\prime}}{g-1} \text {. }
$$

If $\phi \equiv 0$, we get on integration $f-1=c(g-1)$, where $c$ is a constant. Since $\bar{N}(r, 0 ; f) \neq S(r, f)$, there exists $z_{o} \in \mathscr{C}$ such that $f\left(z_{o}\right)=g\left(z_{o}\right)=0$. So $c=1$ and hence $f \equiv g$, which is a contradiction. Therefore $\phi \not \equiv 0$.

Since $f$ and $g$ share $(0,1)$, a multiple zero of $f$ is also a multiple zero of $g$ and so it is a zero of $\phi$. Therefore, by the first fundamental theorem, the Milloux theorem $\{$ p. $55[1]\}$ and Lemma 1 we get

$$
\begin{aligned}
\bar{N}(r, 0 ; f \mid \geq 2) & \leq N(r, 0 ; \phi) \\
& \leq N(r, \phi)+m(r, \phi)+O(1) \\
& =N(r, \phi)+S(r, f) .
\end{aligned}
$$

Now the possible poles of $\phi$ occur only at the poles of $f, g$ and the zeros of $f-1, g-1$. Since $f, g$ share $(1, \infty)$ and $(\infty, \infty)$, it follows that $\phi$ has no pole at all. So from above we get

$$
\bar{N}(r, 0 ; f \mid \geq 2)=S(r, f) .
$$

Secondly we show that $N(r, 1 ; f \mid \geq 2)=S(r, f)$. If $N(r, 1 ; f)=S(r, f)$, there is nothing to prove. So we suppose that $N(r, 1 ; f) \neq S(r, f)$. Let

$$
\psi=\frac{f^{\prime}}{f}-\frac{g^{\prime}}{g} \text {. }
$$

If $\psi \equiv 0$ then $f \equiv c g$, where $c$ is a constant. Since $f, g$ share $(1, \infty)$ and $N(r, 1 ; f) \neq S(r, f)$, it follows that $c=1$ and so $f \equiv g$. This is impossible and so $\psi \not \equiv 0$. 
Since $f$ and $g$ share $(1, \infty)$, it follows that a zero of $f-1$ with multiplicity $m(\geq 2)$ is also a zero of $g-1$ with multiplicity $m(\geq 2)$ and so it is a zero of $\psi$ with multiplicity $m-1$. So by the first fundamental theorem, the Milloux theorem $\{$ p. $55[1]\}$ and Lemma 1 we get

$$
\begin{aligned}
N(r, 1 ; f \mid \geq 2) & \leq 2 N(r, 0 ; \psi) \\
& \leq 2 N(r, \psi)+2 m(r, \psi)+O(1) \\
& =2 N(r, \psi)+S(r, f) .
\end{aligned}
$$

If $f, g$ share $(b, 0)$, we denote by $\bar{N}_{*}(r, b ; f, g)$ the reduced counting function of those $b$-points of $f$ whose multiplicities are different from the multiplicities of the corresponding $b$-points of $g$.

Since $f, g$ share $(0,1)$ and $(\infty, \infty)$, it follows that poles of $\psi$ occur only at those zeros of $f$ whose multiplicities are different from the multiplicities of the corresponding zeros of $g$. Since $\psi$ has only simple poles and $f, g$ share $(0,1)$, it follows from above that

$$
\begin{aligned}
N(r, 1 ; f \mid \geq 2) & \leq 2 \bar{N}(r, \psi)+S(r, f) \\
& \leq 2 \bar{N}_{*}(r, 0 ; f, g)+S(r, f) \\
& \leq 2 \bar{N}(r, 0 ; f \mid \geq 2)+S(r, f) \\
& =S(r, f) .
\end{aligned}
$$

Let $F=f /(f-1)$ and $G=g /(g-1)$. Then $F, G$ share $(0,1),(1, \infty)$ and $(\infty, \infty)$. So by above we get $N(r, 1 ; F \mid \geq 2)=S(r, F)$ and hence $N(r, \infty ; f \mid \geq 2)=$ $S(r, f)$. This proves the lemma.

LEMma 3. If $\alpha$ is a nonconstant entire function then

$$
T\left(r, \alpha^{(p)}\right)=S\left(r, e^{\alpha}\right),
$$

where $\alpha^{(p)}$ is the $p^{\text {th }}$ derivative of $\alpha$.

Proof. Since by the Milloux theorem \{p. $55[1]\}$ and by a result of Clunie \{p. $54[1]\}$ we get

$$
T\left(r, \alpha^{(p)}\right) \leq(p+1) T(r, \alpha)+S(r, \alpha)
$$

and

$$
T(r, \alpha)=S\left(r, e^{\alpha}\right)
$$

the lemma is proved.

Lemma 4. If $f$ and $g$ share $(0,1),(1, \infty),(\infty, \infty)$ and $f \not \equiv g$ then

$$
\frac{f-1}{g-1}=e^{\alpha}
$$


and

$$
\frac{g}{f}=h,
$$

where $\alpha$ is an entire function and $h$ is a meromorphic function with $\bar{N}(r, 0 ; h)=$ $S(r, f)$ and $\bar{N}(r, \infty ; h)=S(r, f)$.

Proof. Since $f$ and $g$ share $(1, \infty),(\infty, \infty)$, it follows that $(f-1) /(g-1)$ has no zero and pole. So there exists an entire function $\alpha=\alpha(z)$ such that

$$
\frac{f-1}{g-1}=e^{\alpha}
$$

Now we put

$$
h=\frac{g}{f} .
$$

Then $h$ is meromorphic and we show that $\bar{N}(r, 0 ; h)=S(r, f)$ and $\bar{N}(r, \infty ; h)=$ $S(r, f)$.

Since $f$ and $g$ share $(0,1),(\infty, \infty)$, it follows that $h$ has a zero at $z_{o}$ if $z_{o}$ is a zero of $f$ and $g$ with multiplicities $m$ and $n$ respectively such that $m<n$; and $h$ has a pole at $z_{o}$ if $n<m$.

Since $f$ and $g$ share $(0,1)$, it follows by Lemma 2 that

$$
\bar{N}(r, 0 ; h) \leq \bar{N}(r, 0 ; g \mid \geq 2)=S(r, f)
$$

and

$$
\bar{N}(r, \infty ; h) \leq \bar{N}(r, 0 ; f \mid \geq 2)=S(r, f) .
$$

This proves the lemma.

Lemma 5. If $f$ and $g$ share $(0,1),(1, \infty),(\infty, \infty)$ and $f \not \equiv g$ then for any $a(\neq 0,1, \infty)$

$$
\bar{N}(r, a ; f \mid \geq 3)=S(r, f) .
$$

Proof. From (1) and (2) we see that

$$
f=\frac{1-e^{\alpha}}{1-h e^{\alpha}}
$$

and so

$$
f-a=\frac{(1-a)+e^{\alpha}(a h-1)}{1-h e^{\alpha}} .
$$

First we suppose that $\alpha$ is nonconstant. If $z_{o}$ is a zero of $f-a$ with multiplicity $\geq 3$ then $z_{o}$ is a zero of

$$
\frac{d}{d z}\left[(1-a)+e^{\alpha}(a h-1)\right]=\alpha^{\prime} e^{\alpha}\left[a h-1+a \frac{h^{\prime}}{\alpha^{\prime}}\right]
$$


with multiplicity $\geq 2$. So $z_{o}$ is a zero of $\alpha^{\prime}$ or $z_{o}$ is a zero of

Therefore

$$
\frac{d}{d z}\left[a h-1+a \frac{h^{\prime}}{\alpha^{\prime}}\right]=a h\left[\frac{h^{\prime}}{h}-\frac{\alpha^{\prime \prime}}{\left(\alpha^{\prime}\right)^{2}} \cdot \frac{h^{\prime}}{h}+\frac{1}{\alpha^{\prime}} \cdot \frac{h^{\prime \prime}}{h}\right] .
$$

$$
\begin{aligned}
\bar{N}(r, a ; f \mid \geq 3) & \leq N\left(r, 0 ; \alpha^{\prime}\right)+\bar{N}(r, 0 ; h)+T\left(r, \frac{h^{\prime}}{h}-\frac{h^{\prime}}{h} \cdot \frac{\alpha^{\prime \prime}}{\left(\alpha^{\prime}\right)^{2}}+\frac{1}{\alpha^{\prime}} \cdot \frac{h^{\prime \prime}}{h}\right) \\
& \leq \bar{N}(r, 0 ; h)+2 T\left(r, \frac{h^{\prime}}{h}\right)+T\left(r, \alpha^{\prime \prime}\right)+4 T\left(r, \alpha^{\prime}\right)+T\left(r, \frac{h^{\prime \prime}}{h}\right)+O(1) .
\end{aligned}
$$

Since by (1), (2), Lemma 1 and Lemma $3 T\left(r, \alpha^{\prime}\right)=S(r, f), T\left(r, \alpha^{\prime \prime}\right)=S(r, f)$, $S(r, h)=S(r, f)$ and by Lemma $4 \bar{N}(r, 0 ; h)=S(r, f), \bar{N}(r, \infty ; h)=S(r, f)$, it follows by the Milloux theorem \{p. $55[1]\}$ that

$$
\begin{aligned}
\bar{N}(r, a ; f \mid \geq 3) & \leq N\left(r, \frac{h^{\prime}}{h}\right)+N\left(r, \frac{h^{\prime \prime}}{h}\right)+S(r, f) \\
& \leq 4 \bar{N}(r, 0 ; h)+4 \bar{N}(r, \infty ; h)+S(r, f) \\
& =S(r, f) .
\end{aligned}
$$

Next we suppose that $\alpha$ is a constant. Let $e^{\alpha}=c$, a constant. Since $f$ is nonconstant, it follows that $h$ is nonconstant and we get

$$
f-a=\frac{(1-a)+c(a h-1)}{1-c h} .
$$

If $z_{o}$ is a zero of $f-a$ with multiplicity $\geq 3$ then $z_{o}$ is a zero of

$$
\frac{d}{d z}[(1-a)+c(a h-1)]=a c h^{\prime}=\operatorname{ach}\left(\frac{h^{\prime}}{h}\right)
$$

with multiplicity $\geq 2$. Therefore by Lemma 4 we get

$$
\begin{aligned}
\bar{N}(r, a ; f \mid \geq 3) & \leq \bar{N}(r, 0 ; h)+T\left(r, \frac{h^{\prime}}{h}\right) \\
& =\bar{N}(r, 0 ; h)+N\left(r, \frac{h^{\prime}}{h}\right)+S(r, f) \\
& =2 \bar{N}(r, 0 ; h)+\bar{N}(r, \infty ; h)+S(r, f) \\
& =S(r, f) .
\end{aligned}
$$

This proves the lemma.

Lemma 6 [3]. Let $f_{1}, f_{2}, f_{3}$ be meromorphic functions such that $f_{1}+f_{2}+$ $f_{3} \equiv 1$. If $f_{1}, f_{2}, f_{3}$ are linearly independent then

$$
T\left(r, f_{1}\right) \leq \sum_{i=1}^{3} N_{2}\left(r, 0 ; f_{i}\right)+\max _{1 \leq i, j(i \neq j) \leq 3}\left\{N_{2}\left(r, \infty ; f_{i}\right)+\bar{N}\left(r, \infty ; f_{j}\right)\right\}+S(r),
$$


where $N_{2}\left(r, b ; f_{i}\right)=\bar{N}\left(r, b ; f_{i}\right)+\bar{N}\left(r, b ; f_{i} \mid \geq 2\right)$ for some $b \in \mathscr{C} \cup\{\infty\}$ and $S(r)=$ $\sum_{i=1}^{3} S\left(r, f_{i}\right)$.

Lemma 7 [9]. Let $f_{1}, f_{2}, f_{3}$ be three nonconstant meromrophic functions such that $f_{1}+f_{2}+f_{3} \equiv 1$ and let $g_{1}=-f_{1} / f_{3}, g_{2}=1 / f_{3}$ and $g_{3}=-f_{2} / f_{3}$. If $f_{1}, f_{2}, f_{3}$ are linearly independent then $g_{1}, g_{2}, g_{3}$ are also linearly independent.

Lemma 8. Let $f$ and $g$ be distinct and share $(0,1),(1, \infty)$ and $(\infty, \infty)$. Let

$$
f_{1}=\frac{(f-a)\left(1-h e^{\alpha}\right)}{1-a}, \quad f_{2}=\frac{-a h e^{\alpha}}{1-a} \quad \text { and } f_{3}=\frac{e^{\alpha}}{1-a},
$$

where $a(\neq 0,1, \infty)$ be a complex number and $h$ and $\alpha$ be defined as in Lemma 4 . If $f_{1}, f_{2}, f_{3}$ are linearly independent then

$$
\text { (i) } N(r, 0 ; f \mid \leq 1) \leq N(r, a ; f \mid \leq 2)+S(r, f)
$$

and

$$
\text { (ii) } N(r, 1 ; f \mid \leq 1) \leq N(r, a ; f \mid \leq 2)+S(r, f) .
$$

Proof. Since $(1-a) f_{1} \equiv 1-e^{\alpha}-a\left(1-h e^{\alpha}\right)$, it follows by Lemma 4 that $\bar{N}\left(r, \infty ; f_{1}\right)=S(r, f)$. Also $\bar{N}\left(r, \infty ; f_{2}\right)=S(r, f)$ and $\bar{N}\left(r, \infty ; f_{3}\right) \equiv 0$. First we suppose that $e^{\alpha}$ is nonconstant.

Now by Lemma 4 and Lemma 6 we get

$$
\begin{aligned}
T\left(r, e^{\alpha}\right) & \leq N_{2}\left(r, 0 ; f_{1}\right)+2 \bar{N}\left(r, 0 ; f_{2}\right)+N_{2}\left(r, 0 ; f_{3}\right)+S(r, f) \\
& =N_{2}\left(r, 0 ; f_{1}\right)+2 \bar{N}(r, 0 ; h)+S(r, f) \\
& =N_{2}\left(r, 0 ; f_{1}\right)+S(r, f) .
\end{aligned}
$$

We see that $(1-a) f_{1} \equiv(f-a)\left(1-h e^{\alpha}\right) \equiv 1-e^{\alpha}-a\left(1-h e^{\alpha}\right)$ and $f=$ $\left(1-e^{\alpha}\right) /\left(1-h e^{\alpha}\right)$. So $z_{o}$ will be a possible zero of $f_{1}$ if either $z_{o}$ is a zero of $f-a$ or $z_{o}$ is a common zero of $1-e^{\alpha}$ and $1-h e^{\alpha}$. Therefore

$$
N_{2}\left(r, 0 ; f_{1}\right) \leq N_{2}(r, a ; f)+N\left(r, 0 ; 1-h e^{\alpha}\right)-N(r, \infty ; f) .
$$

So from (3) we get

$$
T\left(r, e^{\alpha}\right) \leq N_{2}(r, a ; f)+N\left(r, 0 ; 1-h e^{\alpha}\right)-N(r, \infty ; f)+S(r, f) .
$$

Since $f=\left(1-e^{\alpha}\right) /\left(1-h e^{\alpha}\right)$, it follows from Lemma 4 , the first fundamental theorem and (4) that

$$
\begin{aligned}
\bar{N}(r, 0 ; f) & \leq N\left(r, 0 ; 1-e^{\alpha}\right)-N\left(r, 0 ; 1-h e^{\alpha}\right)+N(r, \infty ; f)+\bar{N}(r, \infty ; h) \\
& =N\left(r, 1 ; e^{\alpha}\right)-N\left(r, 0 ; 1-h e^{\alpha}\right)+N(r, \infty ; f)+S(r, f) \\
& \leq T\left(r, e^{\alpha}\right)-N\left(r, 0 ; 1-h e^{\alpha}\right)+N(r, \infty ; f)+S(r, f) \\
& \leq N_{2}(r, a ; f)+S(r, f) .
\end{aligned}
$$

Since by Lemma $2 \bar{N}(r, 0 ; f \mid \geq 2)=S(r, f)$, by Lemma $5 \bar{N}(r, a ; f \mid \geq 3)=$ $S(r, f)$ and $N_{2}(r, a ; f)=N(r, a ; f \mid \leq 2)+2 \bar{N}(r, a ; f \mid \geq 3)$, it follows from (5) that 


$$
N(r, 0 ; f \mid \leq 1) \leq N(r, a ; f \mid \leq 2)+S(r, f) .
$$

If $e^{\alpha}$ is a constant, it follows that $\bar{N}(r, 0 ; f)=S(r, f)$ because $f-1 \equiv e^{\alpha}(g-1)$, $f \not \equiv g$ and $f, g$ share $(0,1)$. So (i) is trivially true.

If $h$ is constant then $h \neq 1$ because $f \not \equiv g$. So from

$$
f-1=\frac{(1-h) e^{\alpha}}{1-h e^{\alpha}}
$$

it follows that $\bar{N}(r, 1 ; f)=S(r, f)$. Hence (ii) is obvious. Therefore we suppose that $h$ is nonconstant.

Let $g_{1}=-f_{1} / f_{3}=-e^{-\alpha}(f-a)\left(1-h e^{\alpha}\right), \quad g_{2}=1 / f_{3}=(1-a) e^{-\alpha}$ and $g_{3}=$ $-f_{2} / f_{3}=a h$. Then $g_{1}+g_{2}+g_{3} \equiv 1$ and by Lemma $7 g_{1}, g_{2}, g_{3}$ are linearly independent. Applying Lemma 6 to $g_{1}, g_{2}, g_{3}$ we get

$$
T(r, h) \leq N_{2}(r, a ; f)+N\left(r, 0 ; 1-h e^{\alpha}\right)-N(r, \infty ; f)+S(r, f) .
$$

Since

$$
f-1 \equiv \frac{(1-h) e^{\alpha}}{1-h e^{\alpha}},
$$

it follows from Lemma 4, the first fundamental theorem, Lemma 5, Lemma 2 and (6) that

$$
\begin{aligned}
N(r, 1 ; f \mid \leq 1) & =\bar{N}(r, 1 ; f)+S(r, f) \\
& \leq \bar{N}(r, 1 ; h)-N\left(r, 0 ; 1-h e^{\alpha}\right)+N(r, \infty ; f)+S(r, f) \\
& \leq N_{2}(r, a ; f)+S(r, f) \\
& =N(r, a ; f \mid \leq 2)+S(r, f) .
\end{aligned}
$$

This proves the lemma.

LEMMA 9. Let $f$ and $g$ be nonconstant meromorphic functions such that $a f+b g \equiv c$, where $a, b, c$ are nonzero constants. Then

$$
T(r, f) \leq \bar{N}(r, 0 ; f)+\bar{N}(r, 0 ; g)+\bar{N}(r, \infty ; f)+S(r, f) .
$$

Proof. By the second fundamental theorem we get

$$
\begin{aligned}
T(r, f) & \leq \bar{N}(r, 0 ; f)+\bar{N}(r, c / a ; f)+\bar{N}(r, \infty ; f)+S(r, f) \\
& =\bar{N}(r, 0 ; f)+\bar{N}(r, 0 ; g)+\bar{N}(r, \infty ; f)+S(r, f) .
\end{aligned}
$$

This proves the lemma.

\section{Proof of Theorem 2 and Theorem 3}

Proof of Theorem 2. Let $f_{1}, f_{2}, f_{3}$ be defined as in Lemma 8. Suppose, if possible, $f_{1}, f_{2}, f_{3}$ are linearly independent. Then by the second fundamental theorem, Lemma 2, Lemma 5 and Lemma 8 we get 


$$
\begin{aligned}
2 T(r, f) \leq & \bar{N}(r, 0 ; f)+\bar{N}(r, 1 ; f)+\bar{N}(r, a ; f)+\bar{N}(r, \infty ; f)+S(r, f) \\
= & N(r, 0 ; f \mid \leq 1)+N(r, 1 ; f \mid \leq 1)+N(r, a ; f \mid \leq 2) \\
& +N(r, \infty ; f \mid \leq 1)+S(r, f) \\
\leq & 3 N(r, a ; f \mid \leq 2)+N(r, \infty ; f \mid \leq 1)+S(r, f),
\end{aligned}
$$

which implies

$$
3 \delta_{2)}(a ; f)+\delta_{1)}(\infty ; f) \leq 2 .
$$

This contradicts the given condition. So there exist constants $c_{1}, c_{2}, c_{3}$, not all zero, such that

$$
c_{1} f_{1}+c_{2} f_{2}+c_{3} f_{3} \equiv 0 .
$$

If possible, let $c_{1}=0$. Then from (7) and the definitions of $f_{2}, f_{3}$ it follows that $h$ is a constant. Since $f \not \equiv g$, we see that $h \neq 1$ and so 1 becomes a Picard's exceptional value of $f$ because $f, g$ share $(1, \infty)$ and $g \equiv h f$.

Again since

$$
f \equiv \frac{1}{h}+\frac{h-1}{h\left(1-h e^{\alpha}\right)},
$$

it follows that $1 / h$ is also a Picard's exceptional value of $f$. So by the second fundamental theorem and Lemma 2 we get

$$
T(r, f) \leq N(r, \infty ; f \mid \leq 1)+S(r, f),
$$

which implies $\delta_{1)}(\infty ; f)=0$. This contradicts the given condition. So $c_{1} \neq 0$.

Also we see that

$$
f_{1}+f_{2}+f_{3} \equiv 1 .
$$

Eliminating $f_{1}$ from (7) and (8) we get

$$
c f_{2}+d f_{3} \equiv 1,
$$

where $c, d$ are constants and $|c|+|d| \neq 0$.

Now we consider the following cases.

CASE I. Let $c \neq 0$ and $d \neq 0$. Then from (9) we get

$$
\frac{-a c h e^{\alpha}}{1-a}+\frac{d e^{\alpha}}{1-a} \equiv 1 \text {. }
$$

If one of $h e^{\alpha}$ and $e^{\alpha}$ is constant then from (10) it follows that the other is also constant and from (1) and (2) we see that $f$ becomes a constant, which is impossible. So $h e^{\alpha}$ and $e^{\alpha}$ are nonconstant.

From (10) we get by Lemma 9 and Lemma 4 that 


$$
\begin{aligned}
T\left(r, e^{\alpha}\right) & \leq \bar{N}\left(r, 0 ; e^{\alpha}\right)+\bar{N}(r, 0 ; h)+\bar{N}\left(r, \infty ; e^{\alpha}\right)+S\left(r, e^{\alpha}\right) \\
& =S(r, f)+S\left(r, e^{\alpha}\right) .
\end{aligned}
$$

Again from (10) we get

$$
d-a c h \equiv \frac{1-a}{e^{\alpha}} .
$$

This implies that $\bar{N}(r, d / a c ; h) \equiv 0$ and $\bar{N}(r, \infty ; h) \equiv 0$. So by the second fundamental theorem we get in view of Lemma 4

$$
\begin{aligned}
T(r, h) & \leq \bar{N}(r, 0 ; h)+\bar{N}(r, d / a c ; h)+\bar{N}(r, \infty ; h)+S(r, h) \\
& =S(r, f)+S(r, h) .
\end{aligned}
$$

Since

$$
f \equiv \frac{1-e^{\alpha}}{1-h e^{\alpha}}
$$

it follows that

$$
T(r, f)=O\left(T\left(r, e^{\alpha}\right)\right)+O(T(r, h)) .
$$

From (11), (12) and (13) we see that there exists a sequence of values of $r$ tending to infinity for which $T(r, f)=o\{T(r, f)\}$. This is a contradiction.

CASE II. Let $c=0$ but $d \neq 0$. From (9) we see that $e^{\alpha}$ is a constant. Since $f \not \equiv g$, it follows from (1) that $e^{\alpha} \neq 1$. So it again follows from (1) that $\bar{N}(r, 0 ; f) \equiv 0$ because $f, g$ share $(0,1)$. Also from (1) and (2) we get

$$
f \equiv \frac{1-e^{\alpha}}{1-h e^{\alpha}} .
$$

By the second fundamental theorem, Lemma 2 and Lemma 4 we get

$$
\begin{aligned}
T(r, f) & \leq \bar{N}(r, 0 ; f)+\bar{N}\left(r, 1-e^{\alpha} ; f\right)+\bar{N}(r, \infty ; f)+S(r, f) \\
& \leq \bar{N}(r, 0 ; h)+N(r, \infty ; f \mid \leq 1)+S(r, f) \\
& =N(r, \infty ; f \mid \leq 1)+S(r, f),
\end{aligned}
$$

which implies that $\delta_{1)}(\infty ; f)=0$. This contradicts the given condition.

CASE III. Let $c \neq 0$ and $d=0$. Then from (9) we see that $h e^{\alpha}=p$, a constant, say. Then $p \neq 1$ because $f \not \equiv g$. So we get

$$
f-a \equiv \frac{(1-a+a p)-e^{\alpha}}{1-p} .
$$


From (14) we see that $T(r, f)=T\left(r, e^{\alpha}\right)+O(1)$. If $1-a+a p \neq 0$, it follows from (14) and Lemma 3 that

$$
N(r, a ; f \mid \geq 2) \leq 2 N\left(r, 0 ; \alpha^{\prime}\right) \leq 2 T\left(r, \alpha^{\prime}\right)=S\left(r, e^{\alpha}\right) .
$$

Hence

$$
\begin{aligned}
N(r, a ; f \mid \leq 2) & =N(r, a ; f)+S(r, f) \\
& =N\left(r, 1-a+a p ; e^{\alpha}\right)+S(r, f) \\
& =T\left(r, e^{\alpha}\right)+S(r, f) \\
& =T(r, f)+S(r, f) .
\end{aligned}
$$

This implies that $\delta_{2)}(a ; f)=0$, which contradicts the given condition.

Therefore $1-a+a p=0$ i.e. $p=(a-1) / a$. Hence from (14) we get

$$
f-a \equiv-a e^{\alpha} .
$$

Also from (2) and (15) we get

$$
g+a-1 \equiv \frac{a-1}{e^{\alpha}} .
$$

From (15) and (16) we obtain

$$
(f-a)(g+a-1) \equiv a(1-a) .
$$

This proves the theorem.

Proof of Theorem 3. Let $F=1-f$ and $G=1-g$. Then $F, G$ are distinct and share $(0,1),(1, \infty),(\infty, \infty)$. Also $\delta_{2)}(1-a ; F)=\delta_{2)}(a ; f)$ and $\delta_{1)}(\infty ; F)=$ $\delta_{1)}(\infty ; f)$. So by Theorem 2 we get

$$
(F-1+a)(G-a) \equiv a(1-a)
$$

i.e.

$$
(f-a)(g+a-1) \equiv a(1-a) .
$$

This proves the theorem.

\section{Application} result.

As an application of Theorem 2 and Theorem 3 we prove the following

THeORem 4. Let $a$ and $b(\neq 0,1)$ be two finite complex numbers and $S_{1}=$ $\left\{a+\alpha: \alpha^{n}+b=0\right\}, S_{2}=\left\{a+\beta: \beta^{n}+b=1\right\}, S_{3}=\{\infty\}$ where $n(\geq 3)$ be $a$ positive integer. If either

$$
E_{f}\left(S_{1}, 1\right)=E_{g}\left(S_{1}, 1\right), \quad E_{f}\left(S_{2}, \infty\right)=E_{g}\left(S_{2}, \infty\right), \quad E_{f}\left(S_{3}, \infty\right)=E_{g}\left(S_{3}, \infty\right)
$$


or

$$
E_{f}\left(S_{1}, \infty\right)=E_{g}\left(S_{1}, \infty\right), \quad E_{f}\left(S_{2}, 1\right)=E_{g}\left(S_{2}, 1\right), \quad E_{f}\left(S_{3}, \infty\right)=E_{g}\left(S_{3}, \infty\right)
$$

then one of the following holds:

$$
\text { (i) } f-a \equiv t(g-a) \text { where } t^{n}=1
$$

and

$$
\text { (ii) }(f-a)(g-a) \equiv s \text { where } 4 s^{n}=1 \text {. }
$$

Proof. We suppose that $E_{f}\left(S_{1}, 1\right)=E_{g}\left(S_{1}, 1\right), \quad E_{f}\left(S_{2}, \infty\right)=E_{g}\left(S_{2}, \infty\right)$, $E_{f}\left(S_{3}, \infty\right)=E_{g}\left(S_{3}, \infty\right)$ because for the other case the theorem can be proved similarly using Theorem 3 .

Let $F=(f-a)^{n}+b$ and $G=(g-a)^{n}+b$. If $F \equiv G$ then case (i) holds. Let $F \not \equiv G$. Clearly $\delta_{2)}(b ; F)=1$ and $\delta_{1)}(\infty ; F)=1$. Since $F, G$ share $(0,1)$, $(1, \infty),(\infty, \infty)$, it follows from Theorem 2 that

$$
(F-b)(G+b-1) \equiv b(1-b)
$$

i.e.

$$
(f-a)^{n}\left\{(g-a)^{n}+2 b-1\right\} \equiv b(1-b) .
$$

From (17) we see that $\infty$ and $a+\sqrt[n]{(1-2 b)}$ are Picard's exceptional values of $g$ where $n \geq 3$, but this is impossible unless $1-2 b=0$. So from (17) we get $(f-a)(g-a) \equiv s$. This proves the theorem.

Acknowledgement. The author is thankful to the referee for his/her valuable suggestions.

\section{REFERENCES}

[ 1] W. K. Hayman, Meromorphic Functions, Oxford Math. Monogr., Clarendon Press, Oxford, 1964.

[2] X. Hua AND M. FAng, Meromorphic functions sharing four small functions, Indian J. Pure Appl. Math., 28 (1997), 797-811.

[ 3 ] I. LAHIRI, Uniqueness of meromorphic functions when two linear differential polynomials share the same 1-points, Ann. Polon. Math., 71 (1999), 113-128.

[4] I. LAHIRI, Weighted sharing and uniqueness of meromorphic functions, Nagoya Math. J., 161 (2001), 193-206.

[5] I. LAHIRI, Weighted value sharing and uniqueness of meromorphic functions, to appear in Complex Variables.

[6] H. Ueda, Unicity theorems for meromorphic or entire functions, Kodai Math. J., 3 (1980), 457-471.

[ 7] S. Z. YE, Uniqueness of meromorphic functions that share three values, Kodai Math. J., 15 (1992), 236-243.

[8] H. X. YI, Meromorphic functions that share three values, Chinese Ann. Math. Ser. A, 9 (1988), 434-439. 
[ 9 ] H. X. YI, Meromorphic functions that share two or three values, Kodai Math. J., 13 (1990), $363-372$.

[10] H. X. YI, Unicity theorems for meromorphic functions that share three values, Kodai Math. J., 18 (1995), 300-314.

Department of Mathematics

UNIVERSITY OF KALYANI

West Bengal 741235

INDIA

e-mail: indrajit@cal2.vsnl.net.in 